\title{
Wax Crystallization from Solution in Hierarchical Morphology Templated by Random Poly(ethylene-co-butene) Self-assemblies
}

\author{
Aurel Radulescu,* Dietmar Schwahn, Jörg Stellbrink, Emmanuel Kentzinger, \\ Manfred Heiderich, and Dieter Richter
}

Institute for Solid State Research, Research Center, Jülich GmbH, 52425 Jülich, Germany

\section{Lewis J. Fetters}

School of Chemical and Biomolecular Engineering, Cornell University, Ithaca, New York 14853-5021

Received May 17, 2006; Revised Manuscript Received July 3, 2006

\begin{abstract}
Semicrystalline poly(ethylene-butene) (PEB- $n$ ) random copolymers can modify the sizes of wax crystals and thus improve the viscoelastic properties of crude oil and middle distillate fuels at low temperatures. The wax-copolymer interactions in solution are rather complex and not fully understood. When the copolymer self-assembles at temperatures higher than the wax cloud point a hierarchy of morphologies templated by the primordial polymer structure evolves with decreasing temperature. Multilevel structures showing characteristic sizes from $10 \mu \mathrm{m}$ to $1 \mathrm{~nm}$ are formed upon cooling in solutions of semicrystalline PEB-7.5 copolymers and in wax containing mixed solutions. The coplymer structures with the different wax molecules were evaluated by three SANS techniques (conventional SANS, focusing-SANS and ultra-SANS) and contrast matching. Complementary microscopy techniques allowed a direct visualization of these structures in real space.
\end{abstract}

\section{Introduction}

Crude oils and refined middle distillate products such as diesel fuel, kerosene or heating oil contain a fraction of paraffins with a broad molecule length distribution. ${ }^{1}$ Depending on the type of deposits or refining technology applied this "wax" fraction will vary between 10 and $30 \mathrm{wt} \%$. $^{2,3}$ These systems undergo a degradation of viscoelastic properties upon cooling below ambient temperature because of the assembly and precipitation of long chain paraffins (or waxes) in platelike crystals with sizes of hundreds of micrometers and an overall morphology resembling a "house-of-cards". ${ }^{4}$ This causes a reduction of material fluidity and a loss of filterability leading to technical problems related to the plugging of pumps, pipelines and engine filters. The pour point $(\mathrm{PP})$-representing the temperature at which the system gels and becomes mechanically rigid ${ }^{2}$-is about $10^{\circ} \mathrm{C}$ for a typical untreated crude oil. ${ }^{1}$ In the case of untreated diesel fuels it may vary over a wide temperature range below $0{ }^{\circ} \mathrm{C}$. A parameter that directly correlates to technical problems motivated by wax crystals is the cold filter plugging point (CFPP). This parameter corresponds to the temperature when plugging occurs in a $45 \mu \mathrm{m}$ filter under standardized conditions. For untreated diesel fuels, the CFPP temperature is normally a few degrees higher than the PP. ${ }^{2}$ To circumvent these technical problems chemical additives are needed.

Those additives that modify the size and shape of the wax crystals appearing in cooled refined fuels are called "pour point depressants". They can consist of semicrystalline polymers that have the capacity to self-assemble in solution without subsequent precipitation even to temperatures of $\sim 253 \mathrm{~K}$. These crystalline-amorphous additives can assume various morphologies but share in common the ability to provide nucleation sites for wax crystallites. These additives must commence to function prior to the fuel reaching its cloud point $(\mathrm{CP})$ : the temperature at which the wax commences to crystallize. ${ }^{2,5}$

To minimize this cold temperature filter plugging problem diesel fuel was initially diluted by kerosene. This is neither

\footnotetext{
* Corresponding author. E-mail: a.radulescu@fz-juelich.de.
}

economically astute nor environmentally wise. ESSO was the first company to develop a practical wax-crystal modifier, in this case from the well-known and long practiced high-pressure free radical copolymerization of ethylene and vinyl acetate (EVA). A crystalline-amorphous diblock copolymer has been introduced $^{6-9}$ as a wax-crystal modifier. SANS measurements revealed the self-assembly of the semicrystalline segment (polyethylene) yielded platelike assemblies stabilized in solution via the amorphous (polyethylene-co-propylene) segments. This "hairy plate" assembly provides an efficient scaffold for moderate sized wax crystal formation hence preventing wax associating into large-scale filter plugging structures.

Recent SANS, ${ }^{10-14}$ rheological, ${ }^{13-15}$ microscopy, ${ }^{14,15}$ and calorimetric $^{15}$ studies have shown that the poly(ethylenebutene) random copolymers cocrystallize with waxes in thin platelets and thus, are very efficient in modifying the wax crystals. These copolymers designated PEB- $n$ ( $n$ is the number of the ethyl side branches/100 backbone carbons) consist of microcrystalline ethylene units copolymerized with amorphous butene segments. ${ }^{15}$ They self-assemble in solution at temperatures that decrease with increasing $n$ and form needlelike structures. ${ }^{12}$ Thus, crystallinity emerges that is tunable by $n$, i.e., the lower $n$ the higher the crystallization tendency and the higher the temperature at which it initially occurs.

In this work we continue the PEB- $n$ study ${ }^{12,14}$ of structures formed upon cooling in solutions and in their common solutions with paraffin waxes for the case of the polymer self-assembling points higher than the cloud-point of the waxes. We combined three small-angle neutron scattering techniques-the conventional pinhole SANS, the focusing-SANS (FSANS), and the ultra-SANS (USANS) double-crystal diffractometry. This allows analysis of the morphologies from nanometer to micrometer sizes. By applying contrast matching a separate analysis of the wax and copolymer conformation within the common morphologies could be studied. The overall morphology of the waxcopolymer common aggregates on a micrometric scale was also investigated by optical and transmission electron microscopy as complementary techniques for the USANS investigations. 
Table 1. Molecular Characteristics and Scattering Properties of the Chemicals Investigated

\begin{tabular}{lllllllll}
\hline & $\mathrm{h}$ PEB-7.5 & $\mathrm{d}$ PEB-7.5 & $\mathrm{C}_{24} \mathrm{H}_{50}$ & $\mathrm{C}_{24} \mathrm{D}_{50}$ & $\mathrm{C}_{36} \mathrm{H}_{74}$ & $\mathrm{C}_{36} \mathrm{D}_{74}$ & $\mathrm{~h}-22$ & $\mathrm{~d}-22$ \\
\hline$M_{\mathrm{w}}\left(\mathrm{g} \mathrm{mol}^{-1}\right)$ & 6000 & 6000 & 338 & 388 & 506 & 580 & 142 & 164 \\
$M_{\mathrm{w}} / M_{\mathrm{n}}$ & 1.02 & 1.02 & 1 & 1 & 1 & 1 & 1 \\
$\xi\left(\mathrm{g} \mathrm{cm}^{-3}\right)$ & 0.863 & 0.986 & 0.8 & 0.92 & 0.8 & 0.92 & 0.73 \\
$M_{0}\left(\mathrm{~g} \mathrm{~mol}^{-1}\right)$ & 56 & 64 & 338 & 388 & 506 & 580 & 142 \\
$V_{0}\left(\mathrm{~g} \mathrm{~mol}^{-1}\right)$ & 64.89 & 64.89 & 422.5 & 422.5 & 632.5 & 632.5 & 194.5 & 164 \\
$\rho\left(10^{10} \mathrm{~cm}^{-2}\right)$ & -0.31 & 7.42 & -0.39 & 7.04 & -0.36 & 7.00 & -0.49 & 6.58
\end{tabular}

Table 2. Samples Investigated with SANS and Microscopy

\begin{tabular}{|c|c|c|c|c|c|c|c|c|c|}
\hline \multirow{2}{*}{$\begin{array}{l}\text { sample no., } \\
\text { contrast }\end{array}$} & \multicolumn{3}{|c|}{ PEB-7.5 } & \multicolumn{2}{|c|}{$\mathrm{C}_{24}$ wax } & \multicolumn{2}{|c|}{$\mathrm{C}_{36}$ wax } & \multirow[b]{2}{*}{ solvent } & \multirow{2}{*}{$\begin{array}{l}\text { instrumental } \\
\text { conditions }\end{array}$} \\
\hline & $h, \Phi_{\mathrm{pol}}$ & $d, \Phi_{\mathrm{pol}}$ & $\mathrm{M}_{\mathrm{W}}$ & $h, \Phi_{\text {wax }}$ & $d, \Phi_{\mathrm{wax}}$ & $h, \Phi_{\text {wax }}$ & $d, \Phi_{\mathrm{wax}}$ & & \\
\hline 1, & $1 \%$ & & 6000 & & & & & $d-22$ & $\begin{array}{l}\mathrm{KWS}{ }^{a}{ }^{a} \mathrm{KWS}^{b} \\
O=\left(1 \times 10^{-4} \div 0.14 \AA^{-1}\right)\end{array}$ \\
\hline 2, polymer visible & $1 \%$ & & 30000 & & & & & $d-22$ & $\begin{array}{l}\mathrm{KWS} 2,{ }^{a} \mathrm{KWS}^{b} \\
Q=\left(1 \times 10^{-4} \div 0.14 \AA^{-1}\right)\end{array}$ \\
\hline polymer visible & $0.6 \%$ & & 6000 & & $4 \%$ & & & $d-22$ & $\begin{array}{l}\mathrm{KWS} 2{ }^{a}{ }^{a} \mathrm{KWS}^{b} \\
Q=\left(1 \times 10^{-4} \div 0.14 \AA^{-1}\right)\end{array}$ \\
\hline wax visible & & $0.6 \%$ & 6000 & $4 \%$ & & & & $\mathrm{~d}-22$ & $\begin{array}{l}\mathrm{KWS} 2,{ }^{a}{ }^{\mathrm{KWS}} 3,{ }^{b} \mathrm{DKD}^{b} \\
Q=\left(3 \times 10^{-5} \div 0.14 \AA^{-1}\right)\end{array}$ \\
\hline 5, & $0.6 \%$ & & 6000 & & $0.5 \%$ & & & $d-22$ & $\begin{array}{l}\mathrm{KWS} 2^{a} \\
Q=\left(2 \times 10^{-3} \div 0.14 \AA^{-1}\right)\end{array}$ \\
\hline 6, wax visible & & $0.6 \%$ & 6000 & $0.5 \%$ & & & & $d-22$ & $\begin{array}{l}\mathrm{KWS} 2,{ }^{a}{ }^{\mathrm{KWS}} 3{ }^{b}{ }^{b} \mathrm{DKD}^{b} \\
Q=\left(3 \times 10^{-5} \div 0.14 \AA^{-1}\right)\end{array}$ \\
\hline 6, & $1 \%$ & & 30000 & & & & $4 \%$ & $d-22$ & $\begin{array}{l}\mathrm{KWS} 2,^{b} \mathrm{KWS}^{b} \\
Q=\left(1 \times 10^{-4} \div 0.14 \AA^{-1}\right)\end{array}$ \\
\hline 7, wax visible & $1 \%$ & & 30000 & & & & $4 \%$ & h-22 & $\begin{array}{l}\mathrm{KWS} 2^{b} \\
Q=\left(2 \times 10^{-3} \div 0.14 \AA^{-1}\right)\end{array}$ \\
\hline
\end{tabular}

$h$ and $d$ define the hydrogenated or the deuterated status of the components; $a$ and $b$ represent either the older conventional SANS measurements ${ }^{14}$ or the present investigations.

\section{Experimental Section: Materials and Methods}

2.1. Samples. The PEB-7.5 copolymers have been synthesized in cyclohexane via the anionic polymerization of 1,4-butadiene ${ }^{16}$ with tert-butyllithium used as initiator. The vinyl content was controlled by the addition of triethylamine as a modifier of the chain microstructure. After hydration, the vinyl units led to the ethyl side branches of the otherwise linear PE. The ethyl side branch content was about 7.5 per 100 carbon atoms in the main chain, as determined by H NMR. Copolymers with two molecular weights $\left(M_{\mathrm{w}}\right)$ were synthesized, namely 6 and $30 \mathrm{~kg} / \mathrm{mol}$. The copolymer with the lower $M_{\mathrm{w}}$ was obtained in both hydrogenated and deuterated states while the higher $M_{\mathrm{w}}$ material was produced only in the hydrogenated format.

The self-assembling properties in solution were investigated with FSANS and SANS in order to determine the polymer morphologies formed over a wide length scale. For this purpose polymer solutions (volume fraction $\Phi=1 \%$ ) in decane were prepared for each sample. Fully protonated polymers have been dissolved in fully deuterated decane thus obtaining the maximum contrast available for the neutron scattering measurements. The solutions were first heated to $85^{\circ} \mathrm{C}$ in decane and then gradually cooled in order to study the aggregation behavior as a function of decreasing temperature. The wax-copolymer solutions were prepared via mixing of the copolymers with long chain paraffin waxes in decane and were subject of the same thermal treatment as the pure polymer solutions. The wax $\mathrm{CP}$ in hydrocarbon solvents increases with increasing carbon number ${ }^{17}$ while the PEB-7.5 self-assembling point increases with increasing $M_{\mathrm{w}} \cdot{ }^{14}$ This leads to the possibility of combining in common solution different $M_{\mathrm{w}}$ PEB-7.5 copolymers and wax molecules in order to have a certain interaction mechanism within a temperature range of interest. Thus, for the investigation of the wax crystallization templated by the precursor polymeric aggregates we examined the aggregation behavior of polymer-wax mixed solutions obtained from the combination of the low $M_{\mathrm{w}}$ copolymer with a shorter paraffin wax molecule $\left(\mathrm{C}_{24}\right)$ and of high $M_{\mathrm{w}}$ copolymer with a longer paraffin wax molecule $\left(\mathrm{C}_{36}\right)$. In both situations the copolymers self-assemble above the wax $\mathrm{CP}$ and form density modulated 1-dimensional aggregates that later nucleate the wax crystals. ${ }^{13}$ A detailed SANS study of the structures formed by the wax-copolymer interaction upon cooling requires the contrast matching technique in which the degree of hydrogenation and deuteration of different components of the sample is varied in order to allow either the polymer or the wax visible. For this purpose, taking into account the scattering length densities of the protonated and deuterated materials involved in this study (Table 1), we prepared two solutions for each copolymer-wax combination mentioned above. For the $6 \mathrm{~kg} / \mathrm{mol}$ PEB-7.5 and $\mathrm{C}_{24}$ wax, we prepared the solutions with deuterated copolymer/protonated wax/ deuterated solvent for wax visible and with protonated copolymer/ deuterated wax/deuterated solvent for copolymer visible. For the combination of the $30 \mathrm{~kg} / \mathrm{mol} \mathrm{PEB}-7.5$ and $\mathrm{C}_{36}$ wax, we chose solutions with protonated copolymer/deuterated wax/protonated solvent for wax visible and the protonated copolymer/deuterated wax/deuterated solvent for the copolymer visible. For the case of the $\mathrm{C}_{36}$ wax visible the use of protonated solvent led to a higher incoherent background. Nevertheless, since the wax formed large structures whose scattering was very strong, a good analysis within the low $Q$ range and a fair interpretation at high $Q$ after the subtraction of the flat solvent scattering was possible (see section 3.4.).

The PEB-7.5 samples self-assemble in solution above room temperature. The resultant micelles remain in solution to temperatures of at least $253 \mathrm{~K}$ without signs of precipitation. In contrast, samples where $n$ is $<\sim 5$ will precipitate at room temperature, thus rendering those copolymers unfit for these studies. Premature precipitation of a fraction of the material is characteristic of the commercial EVA copolymers. ${ }^{18}$

2.2. Small-Angle Neutron Scattering: Experiments and Data Evaluation. Three types of SANS techniques have been involved in the present exploration. The parameters of the samples studied are presented in Table 2 . The conventional SANS measurements were performed on the KWS2 instrument ${ }^{19}$ at the FRJ-2 research reactor of the Research Center Jülich. The measurements were done with a neutron wavelength of $\lambda=7.2 \AA$ with a spread of $\Delta \lambda / \lambda=$ $10 \%$. Hence, the $Q$ range between 0.002 and $0.14 \AA^{-1}$ was accessible using three sample-to-detector distances, namely $\mathrm{L}=2,8$, and 20 $\mathrm{m}$. The samples containing deuterated solvent were investigated in $2 \mathrm{~mm}$ thick quartz cells while that containing protonated solvent (sample 7) was studied in $1 \mathrm{~mm}$ thick quartz cell in order to avoid low transmission due to incoherent scattering. The data were 
corrected for the scattering from empty cell and calibrated in absolute units by using a Lupolen secondary standard according to

$$
\left(\frac{\mathrm{d} \Sigma}{\mathrm{d} \Omega}(Q)\right)^{\mathrm{s}}=\frac{L^{\mathrm{l}} h^{1} T^{\mathrm{l}} \mathrm{MON}^{1}}{L^{\mathrm{s}} h^{\mathrm{s}} T^{\mathrm{s}} T^{\mathrm{ec}} \mathrm{MON}^{\mathrm{s}}\left\langle I^{\mathrm{l}}\right\rangle}\left(\frac{\mathrm{d} \Sigma}{\mathrm{d} \Omega}(0)\right)^{1}\left(I^{\mathrm{s}}(Q)-T^{\mathrm{s}} I^{\mathrm{ec}}(Q)\right)
$$

Here 1, s, and ec refer to the Lupolen, sample, and empty cell, respectively, $T$ denotes the transmission, MON is the total amount of the monitor counts, $I(Q)$ is the scattered intensity, $h$ is the sample thickness, $L$ is the sample-to-detector distance and $\langle I\rangle$ is the averaged measured intensity of the Lupolen. The value of $\mathrm{d} \Sigma(0) /$ $\mathrm{d} \Omega$ for the Lupolen standard has been calibrated with vanadium to be $1.8155 \mathrm{~cm}^{-1}$. After radial averaging the calculated incoherent contribution of the polymer or wax as well as the measured solvent scattering was subtracted to obtain the coherent macroscopic scattering cross section of the aggregates. Although most of the conventional SANS measurements have been detailed analyzed elsewhere $^{14}$ (see Table 2 ), we will briefly present here these results in order to allow a complete and easier understanding of the complex morphologies formed in solution by the materials under the present investigation.

The KWS3 focusing-mirror SANS diffractometer ${ }^{19,20}$ installed at the FRJ-2 reactor is a unique instrument and works on the principle of one-to-one image of the entrance aperture on a 2-dimensional position sensitive detector via neutron reflection from a double-focusing toroidal mirror with a focal length of $11 \mathrm{~m}$. The entrance pinhole is at one focus while the other focus is in the detection plane of the detector. The sample position is at $1.7 \mathrm{~m}$ after the mirror center. At this place, the beam is $10 \mathrm{~cm}$ wide and $2 \mathrm{~cm}$ high. The neutron intensity integrated over the complete beam cross-section is $180 \mathrm{~ns}^{-1}$ per $\mathrm{mm}^{-2}$ cross section of the entrance aperture. The instrument works with a neutron wavelength $\lambda=$ $12.7 \AA$ with a spread of $\Delta \lambda / \lambda=9 \%$. The typical $Q$ range covered is $1 \times 10^{-4}$ to $1 \times 10^{-3} \AA^{-1}$ but optionally, this can be extended up to $2 \times 10^{-3} \AA^{-1}$ in the case of an off-centered dector position. All the measurements have been done without the beam-stop. Therefore, the sample transmission was directly calculated from the intensity at $Q=0$ with respect to the empty beam. The data have been corrected for the detector efficiency and empty cell contribution and then radially averaged and shifted vertically until they match with those measured in absolute units at the KWS-2.

The USANS measurements have been performed at the DKD double-crystal diffractometer ${ }^{19}$ that can cover a $Q$ range between $2 \times 10^{-5}$ and $10^{-3} \AA^{-1}$. The resolution of the instrument is determined by two perfect single Si crystals, a monochromator and an analyzer one, mounted on an optical bench and installed in a separately shielded room in order to protect the instrument against vibrations and temperature gradients. The sample is situated between the two crystals. To measure the neutrons scattered into an angle $\theta$, the analyzer crystal has to be rotated by the same angle $\theta$ from its position parallel to the monochromator crystal. The background intensity from the Si crystals is considerably reduced by using channel-cut crystals in which the neutrons are reflected several times. The instrument was operated with a neutron wavelength of $4.5 \AA$. The high- $Q$ resolution exists in one dimension only, therefore slit corrections have to be applied. ${ }^{21}$ The slit-smeared cross-section $\mathrm{d} \tilde{\Sigma} / \mathrm{d} \Omega$ is related to scattering cross-section $\mathrm{d} \Sigma / \mathrm{d} \Omega$ obtained at the pinhole instruments by

$$
\frac{\mathrm{d} \tilde{\Sigma}}{\mathrm{d} \Omega}=\frac{1}{\Delta Q_{\mathrm{V}}} \int \frac{\mathrm{d} \Sigma}{\mathrm{d} \Omega}(Q) \mathrm{d} Q_{\mathrm{V}}
$$

where $Q_{\mathrm{V}}$ is the scattering vector in the vertical slit direction. $\mathrm{d} \tilde{\Sigma}$ $/ \mathrm{d} \Omega$ in the absolute units is calculated from the scattering intensity $\Delta I(Q)$ by using the following equation

$$
\frac{\mathrm{d} \tilde{\Sigma}}{\mathrm{d} \Omega}(Q)=\frac{k_{0}^{2} \Delta I(Q)}{I_{0} F D T \Delta Q_{V} \Delta Q_{H}}
$$

where $k_{0}$ is the incident wave vector, $I_{0}$ is the primary intensity, $F$

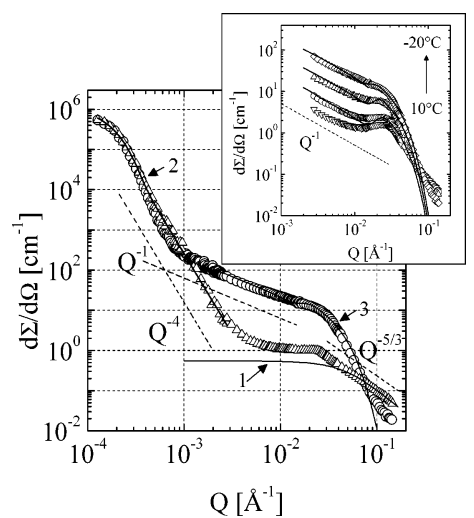

Figure 1. Scattering patterns from a solution of PEB-7.5 copolymer $(6 \mathrm{~K})$ in $\mathrm{d}-22$ (sample 1) at two temperatures within the aggregation regime: +20 (open triangles) and $-20{ }^{\circ} \mathrm{C}$ (open circles). The dotted lines indicate the power law behavior in different $Q$ ranges while the solid lines represent the model description of experimental data within particular $Q$ ranges: 1, the Beaucage form factor of polymer single coils; 2 , the Beaucage form factor of large, compact aggregates; 3 , the density modulated polymer needles. The inset shows the temperature evolution of the correlation peak and $Q^{-1}$ power law behavior: the open symbols are the experimental data while the continuous lines represent the fit according to the density modulated $1 \mathrm{D}$ model. ${ }^{14}$

and $D$ are the area and the thickness of the sample, $T$ is the sample transmission, and $\Delta Q_{\mathrm{V}}$ and $\Delta Q_{\mathrm{H}}$ are the vertical and horizontal momentum resolution, respectively. $\Delta Q_{\mathrm{H}}$ is the half-width of the empty beam, typically around $2 \times 10^{-5} \AA^{-1}$, while $\Delta Q_{\mathrm{V}}$ is about 3 orders of magnitude larger. In this condition, an infinite slit height can be assumed for which the following analytical expression for deconvolution becomes applicable:

$$
\frac{\mathrm{d} \Sigma}{\mathrm{d} \Omega}(Q)=-\frac{\Delta Q_{V}}{\pi} \int_{0}^{\infty} \frac{\frac{\mathrm{d} \tilde{\Sigma}^{\prime}}{\mathrm{d} \Omega}\left(\sqrt{\left(Q^{2}+x^{2}\right)}\right)}{\left(\sqrt{\left(Q^{2}+x^{2}\right)}\right)} \mathrm{d} x
$$

At the KWS3 and DKD instruments the multiple scattering effects become very important and dominate for sample thickness within the range $0.5-2 \mathrm{~mm}$, typical for a conventional SANS experiment. To minimize the contribution of such effects and to have a reasonable transmission (higher than 70\%) for the samples presented in Table 2 and characterized by either the polymer or wax full contrast we varied the thickness between 0.2 and $0.5 \mathrm{~mm}$.

2.3. Microscopy Observations. Several polymer and wax solutions as well as polymer-wax mixed solutions were examined with a Zeiss Axioplan 2 microscope in a transmission light direct geometry. The observations have been performed at different temperatures using a Linkam THMS600 temperature stage. The temperatures of interest have been chosen in correlation with the present FSANS and USANS measurements in order to bring support for the interpretation of the scattering data. The micrographs of the structures and morphologies formed by the investigated materials through self-assembling, crystallization or common aggregation processes were captured with a low-light interacting camera. The images were obtained at different magnifications and the scale bars are shown for the microscopy figures. For the combination of the high $M_{\mathrm{w}}$ PEB-7.5 copolymer with the $\mathrm{C}_{36}$ wax in decane the common aggregates formed were isolated from solution at room temperature on a carbon grid and examined with a CM200 Philips transmission electron microscope (TEM).

\section{Results and Discussions}

3.1. SANS and FSANS from the 6K PEB-7.5 Copolymer. Figure 1 presents the scattered intensity in absolute units of the low $M_{\mathrm{w}}$ PEB-7.5 random copolymer (sample 1 in Table 2) in decane at two temperatures within the aggregation regime. The data obtained by combining the SANS ${ }^{14}$ and FSANS instruments reveal for different size scales rich structural features of the 
polymer aggregates. At $20{ }^{\circ} \mathrm{C}$ the scattering profile at high $Q$ follows a $Q^{-5 / 3}$ behavior which is indicative for single polymer chains in a "good" solvent showing excluded volume interaction. The experimental data within this $Q$ range agree with the Beaucage single coil form factor depicted by the model curve " 1 ". This was calculated following the unified equation ${ }^{22}$

$$
\frac{\mathrm{d} \Sigma}{\mathrm{d} \Omega}(Q)=G \exp \left(-Q^{2} R_{\mathrm{g}}^{2} / 3\right)+B\left\{\left[\operatorname{erf}\left(k Q R_{\mathrm{g}} / \sqrt{6}\right)\right]^{3} / Q\right\}^{P}
$$

for a power law exponent $P=5 / 3$, thereby using the radius of gyration $R_{\mathrm{g}}$ and the "forward scattering" obtained from a Zimm analysis of the scattering data at $85^{\circ} \mathrm{C}$, when all polymers are dissolved in decane. ${ }^{14} G$ is the classical Guinier prefactor which for an ensemble of aggregating structures is defined as $G=$ $N V^{2}(\Delta \rho)^{2}$, with $N$ being the number density and $V$ being the volume of an aggregate, while $B$ is a prefactor specific to the type of power-law regime. The constant $k$ accounts for an approximation involved in the description of the low- $Q$ powerlaw limit ${ }^{22}$ and has a value of 1 for a steep power-law decays $(P>3)$ and deviates slighthly from 1 for a weak power-law decays. Empirically, is found to be close to 1.06 for $P$ between 1.5 and 3 . The evaluation shows that most of the polymer chains are still in solution at $20^{\circ} \mathrm{C}$. At lower $Q$, however, the scattering pattern displays significant deviations from the single coil form factor. Its tendency to increase continuously indicates that the polymers self-assemble to a certain extent yielding larger scale aggregates. A broad peak-like feature appears to evolve at around $Q^{*}=0.025 \AA^{-1}$. This peak relates to preferred correlations with an average length scale of about $250 \AA$. At lower $Q$ the scattered intensity increases dramatically and the scattering features a $Q^{-4}$ power-law behavior extending over a wide $Q$ range and a Guinier-like regime at very low $Q$. This observation shows the formation of compact aggregates with sizes within the micrometer range. The model description in terms of the Beaucage form factor (eq 5) for a power law exponent $P=4$ is depicted by the curve " 2 " and results in a radius of gyration $\cong 1 \mu \mathrm{m}$. The "forward scattering" of these large aggregates amounts to $\mathrm{d} \Sigma(Q=0) / \mathrm{d} \Omega \cong 1.1 \times 10^{6} \mathrm{~cm}^{-1}$. In comparison with that at $20{ }^{\circ} \mathrm{C}$ the scattering pattern at $-20{ }^{\circ} \mathrm{C}$ displays major changes at intermediate and high $Q$ but follows the same profile at low $Q$. Obviously, the big, compact aggregates are not influenced by the temperature decrease. The $T$-independent scattering level shows that these aggregates are not growing in size nor increasing in number and one can conclude that they are already formed and stabilized at $20{ }^{\circ} \mathrm{C}$. At intermediate $Q$, the scattering profile follows a clear $Q^{-1}$ power law while toward high $Q$ it shows a progression to a very broad hump and a step intensity decrease. The power law is indicative for the scattering from needlelike aggregates while the intensity drop at high $Q$ may relate to the form factor of the lateral profile of the polymer needles. The broad hump at around $Q^{*}=0.025$ $\AA^{-1}$ represents the ultimate stage of the temperature evolution of the correlation peak at $20^{\circ} \mathrm{C}$, a process which is presented in detail by the inset of Figure 1. There, the development of the SANS profile from the needlelike aggregates with decreasing temperature are shown. ${ }^{14}$ From these observations we may conclude that the needlelike aggregates and the large compact objects giving rise to the scattering at very low $Q$ represent separate structures which form at different temperatures and develop independently from each other.

The length of the polymer needles is not accessible, since their scattering at low $Q$ is buried by the scattering from the compact objects. However, it ranges to several hundred nanometers. Systematic SANS studies performed for different polymer concentrations at different temperatures ${ }^{14}$ have shown that the observed correlation must arise from a modulation of the density in terms of alternating amorphous and crystalline sequences along the polymer needle. This morphology was characterized in a detailed fashion in terms of a density modulated-1D model. ${ }^{14}$ The model curve " 3 " in Figure 1 and the curves in the inset represent an interpretation of the intermediate and high $Q$ scattering data in terms of this model. ${ }^{14}$

The small discrepancy between the experimental data and the model above $Q=0.1 \AA$ may be related to additional scattering from the polymer chains still in solution.

The SANS ${ }^{14}$ and FSANS studies have definitely established that decreasing the temperature the highly crystalline PEB-7.5 random copolymer in solution displays a two-stage selfassembling process yielding two separate morphologies: (i) the fast formation of large compact aggregates which occurs at high temperature (around $40{ }^{\circ} \mathrm{C}^{14}$ ) and (ii) the gradual evolution of density modulated 1 -dimensional aggregates below $20^{\circ} \mathrm{C}$. The large compact aggregates, which become stable at around room temperature, relate to the faster crystallization of those chains containing long and uninterrupted methylene repeat units. The needle formation starts at around $20^{\circ} \mathrm{C}$ with the occurrence of some crystalline nuclei correlated in a 1-dimensional arrangement by a mechanism which is still not yet understood. The broad correlation peak indicates that the effect is not very strong and the correlation length has a wide distribution. As the temperature decreases, the peak becomes less prominent. That may relate to a continuous filling of the space between the crystalline nuclei with the more highly aggregated polymers.

The two kinds of polymeric aggregates have been elucidated via optical microscopy. Figure 2 presents a series of micrographs collected from sample 1 at room temperature (Figure 2a) and sample 3 at $-10{ }^{\circ} \mathrm{C}$ (Figure $2 \mathrm{~b}$ ) in parallel with that from a pure solution of $4 \% \mathrm{C}_{24}$ wax in decane at $-10^{\circ} \mathrm{C}$ (Figure 2c). First of all, the micrographs allow a direct visualization of the large compact objects formed at higher temperature. A close inspection of these objects (inset of Figure 2a) reveals that they show a dumbbell like shape rather than a spherical one. The model interpretation of the scattering from dumbbell morphology involves a complicated formalism ${ }^{23}$ and could reveal the scattering of neighboring spheres at lower $Q$ values than those explored in the present measurement.

Within the $Q$ range covered by FSANS, the scattering is governed by the individual spheres and thus, a rough characterization of these objects is possible by combining the size and structural features observed in the micrographs with the parameters obtained from the interpretation of the FSANS data with the Beaucage model. ${ }^{22}$ The "forward scattering" of a compact sphere of a diameter of $2 \mu \mathrm{m}$, as revealed by the micrograph, leads to the conclusion that a fraction of the $0.55 \%$ of all polymers reside inside these aggregates. This value is in close agreement with that obtained earlier from the interpretation of the Porod constant observed by the low $Q$ in the SANS profiles. $^{14}$

The micrographs also permitted an indirect visualization of the thin polymeric needles. When in a common solution the PEB-7.5 copolymer is mixed with a paraffin wax $\left(\mathrm{C}_{24}\right)$ with a crystallization point much lower than the polymer self-assembling temperature, then the crystallization event forms the needlelike structures (Figure $2 b$ ). This behavior differs markedly from that shown by the wax alone in solution (Figure 2c). There big, platelike crystals resembling a house-of-cards morphology are formed. The crystallization of wax in 1-dimensional objects (when it is mixed with the random copolymer) is driven by the 
a)

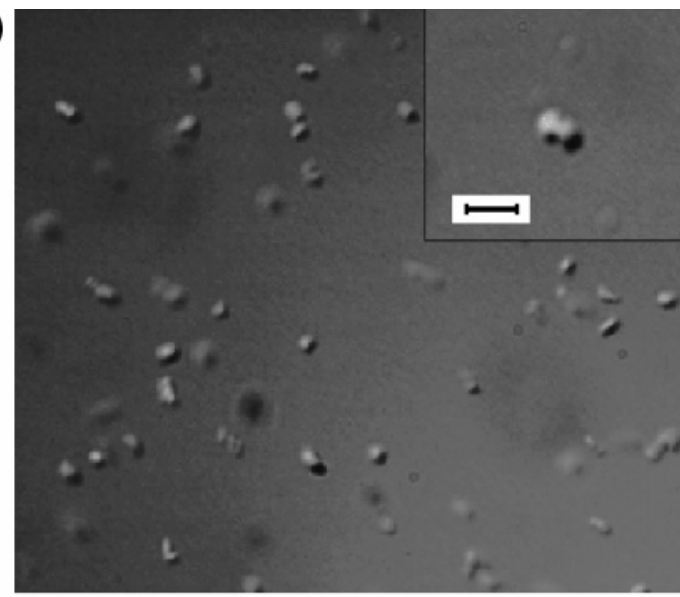

b)

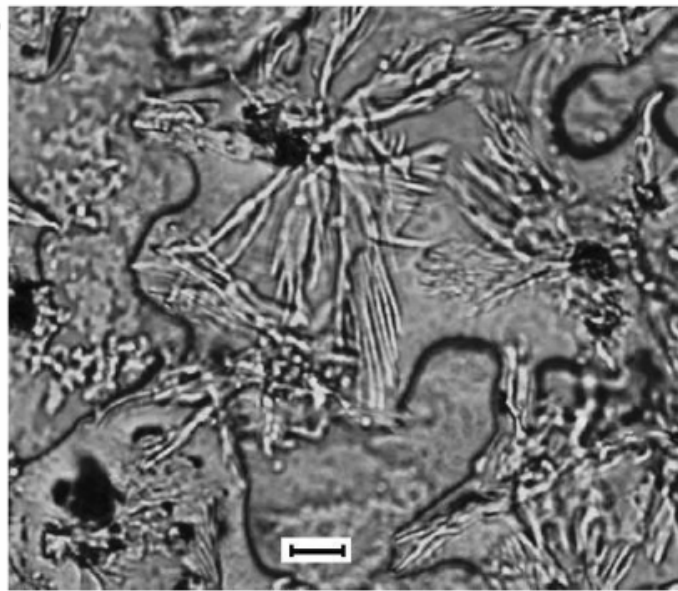

c)

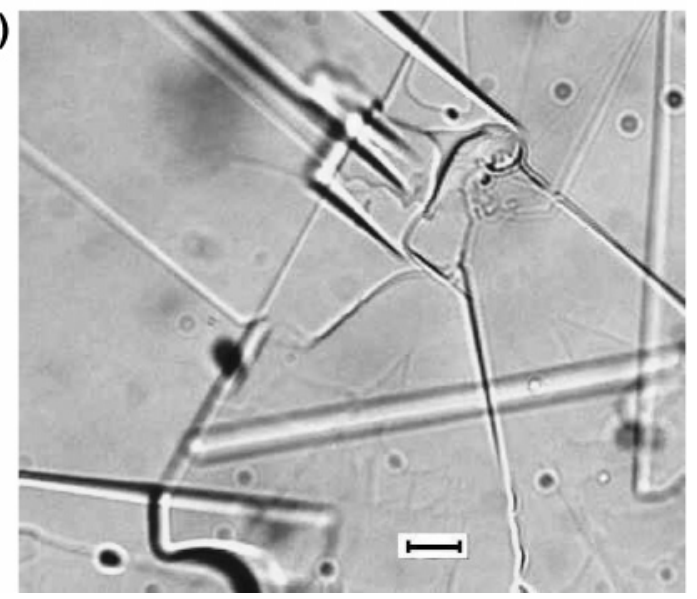

Figure 2. Optical micrographs: (a) from decane solutions 6K PEB7.5 at room temperature (scale bar for the inset $4 \mu \mathrm{m}$ ); (b) from $6 \mathrm{~K}$ PEB-7.5 and $4 \% \mathrm{C}_{24}$ wax at $-10{ }^{\circ} \mathrm{C}$ (scale bar $30 \mu \mathrm{m}$ ); (c) from $4 \%$ $\mathrm{C}_{24}$ wax at $-10^{\circ} \mathrm{C}$ (scale bar $20 \mu \mathrm{m}$ ).

existence of primordial needlelike polymer aggregates which dictate the overall morphology and act as nucleation platforms for the wax crystallites. One can generally say that the polymer aggregates template the wax crystallization. Thus, the polymer needles are decorated by the wax and in this way detected via microscopy.

3.2. SANS and FSANS from the High $M_{\mathrm{w}}$ PEB-7.5 Copolymer. Figure 3 presents the scattering pattern from a solution of the high $M_{\mathrm{w}}$ PEB-7.5 copolymer in decane (sample 2) at room temperature by combining SANS ${ }^{14}$ and FSANS. As observed from the comparison of the scattering profile with a single coil form factor (curve " 1 ") the polymers are seen to

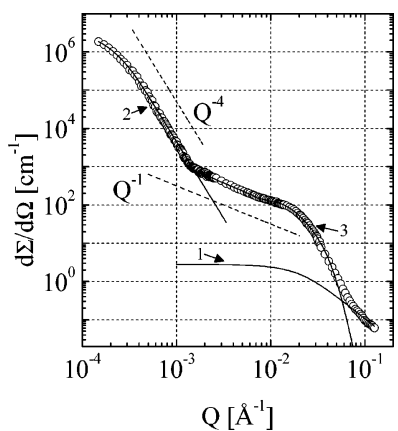

Figure 3. Scattering pattern from a solution of PEB-7.5 copolymer (30K) in d-22 at $20^{\circ} \mathrm{C}$ (sample 2). Lines have the same meaning as in Figure 1.

aggregate. The structural features are similar to those revealed by the solution of low $M_{\mathrm{w}}$ PEB-7.5 in the aggregation regime at $-20{ }^{\circ} \mathrm{C}$. At intermediate $Q$ the scattering pattern indicates the presence of density modulated needles (curve " 3 ") although a clear correlation peak is not visible. The low $Q$ scattering from the large compact aggregates dominates and was described again in terms of the Beaucage model (eq 5) with an exponent $P=4$ (curve "2"). The radius of gyration $R_{\mathrm{g}}$ and the "forward scattering" of these objects could be evaluated. The compact aggregates formed by the high $M_{\mathrm{w}}$ copolymer are roughly of the same size as those formed by the low $M_{\mathrm{w}}$ material. The "forward scattering" $\mathrm{d} \Sigma(Q=0) / \mathrm{d} \Omega \cong 3.4 \times 10^{6} \mathrm{~cm}^{-1}$ interpreted again in terms of compact spheres revealed that these objects contain a higher polymer amount, about $1.7 \%$ of all polymers in solution.

Again, two kinds of polymer aggregates may be observed with the microscope. Parts $\mathrm{a}$ and $\mathrm{b}$ of Figure 4 present micrographs similar to those reported above for the low $M_{\mathrm{w}}$ material, with the difference that all were collected at room temperature. The compact polymer objects that are formed at high temperature by the crystallization of chains containing long uninterrupted methylene sequences show the same dumbbell morphology. The visualization of the polymeric needles was possible after decoration with $\mathrm{C}_{36}$ wax crystals. Principally, the same mechanism of wax needle formation should apply. The high $M_{\mathrm{w}}$ copolymer forms 1-dimensional aggregates showing a longitudinal density modulation while the wax crystallizes only later and nucleates on the polymeric 1-dimensional structures already formed instead of forming big, compact structures arranged in a "house-of-cards" morphology (see Figure 2c).

3.3. Crystallization of $\mathrm{C}_{24}$ Wax from Solution in the Presence of Low $\boldsymbol{M}_{\mathrm{w}}$ PEB-7.5 Copolymer. Figure 5 presents separately the polymer and wax scattering pattern from the mixed solution of low $M_{\mathrm{w}}$ PEB-7.5 copolymer and $4 \% \mathrm{C}_{24}$ wax in d-22 (samples 3 and 4) at two temperatures within the common aggregation regime (well below the temperature of polymer needles appearance). The $Q^{-2}$ power law behavior at intermediate $Q$ and the peak at around $Q^{*}=0.025 \AA^{-1}$ shown at $0{ }^{\circ} \mathrm{C}$ by the scattering from both components reveal a cocrystallization of polymer and wax into thin, very large platelets forming a correlated arrangement. The lateral size of these platelets can be evaluated by analyzing the Guinier regime identified at very low $Q$ by USANS under wax contrast. The USANS data collected in the slit geometry have been transformed to the pinhole geometry according to eq 4 . The structural and density parameters have been obtained by modeling this morphology in terms of the paracrystalline structure factor

$$
S^{\mathrm{int}}(Q)=\frac{\sinh \left(Q^{2} \sigma_{\mathrm{D}}{ }^{2} / 4\right)}{\cosh \left(Q^{2} \sigma_{\mathrm{D}}{ }^{2} / 4\right)-\cos \left(Q D^{*}\right)}
$$




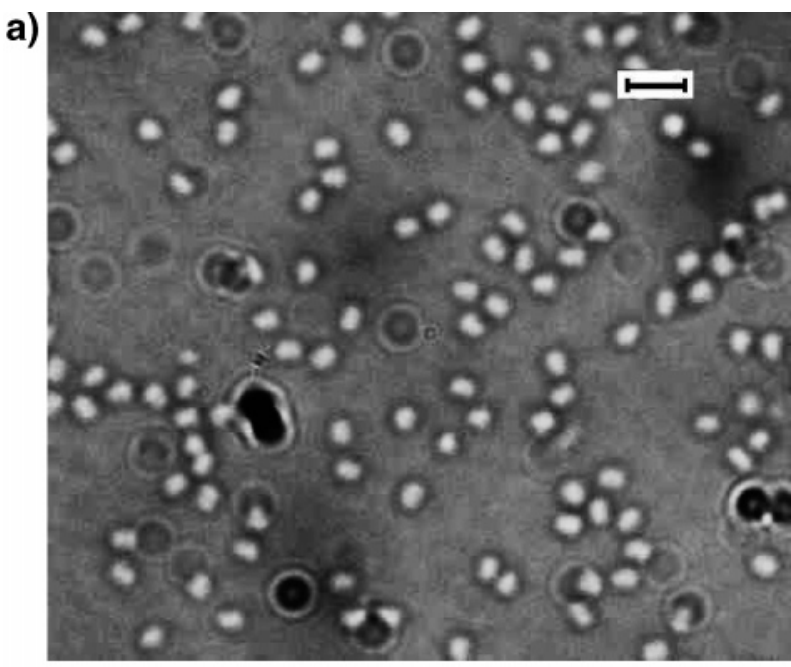

b)

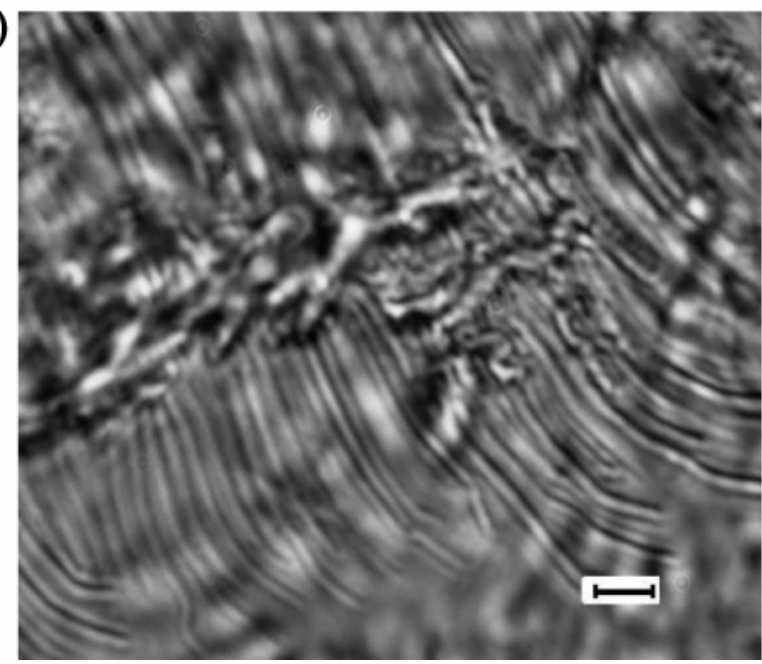

Figure 4. Optical micrographs: (a) from room-temperature decane solutions of 30K PEB-7.5 (scale bar $4 \mu \mathrm{m}$ ); (b) from 30K PEB-7.5 and $4 \% \mathrm{C}_{36}$ wax (scale bar $20 \mu \mathrm{m}$ ).

applied to an ensemble of finite size disks, each disk being made by a wax and a polymer embedded layers

$$
\left.\frac{\mathrm{d} \Sigma}{\mathrm{d} \Omega}(Q)\right|_{p, w}=\frac{\Phi_{\mathrm{p}, \mathrm{w}}^{\mathrm{plt}}}{V_{\mathrm{p}, \mathrm{w}}^{\mathrm{plt}}}\left(\pi R^{2}\right)^{2} P_{\mathrm{plt}}(Q)_{\mathrm{p}, \mathrm{w}} S^{\mathrm{int}}(Q) \frac{D(Q R / 2)}{(Q R / 2)}
$$

an approach similar to that presented in ref 14 . In this case, the layer form factor is

$$
P_{\mathrm{plt}}(Q)_{\mathrm{p}, \mathrm{w}}=\left(\Delta \rho_{\mathrm{p}, \mathrm{w}}^{\mathrm{plt}}\right)^{2} d_{\mathrm{p}, \mathrm{w}}{ }^{2}\left(\frac{\sin \left(Q d_{\mathrm{p}, \mathrm{w}} / 2\right)}{\left(Q d_{\mathrm{p}, \mathrm{w}} / 2\right)}\right)^{2}
$$

In eqs $6-8, \Phi^{\text {plt }}$ is the disk (platelet) volume fraction, $V^{\text {plt }}$ is the volume, $R$ is the radius, $d$ is the thickness, and $\Delta \rho^{\text {plt }}$ is the contrast, while $p$ or $w$ denotes either the polymer or the wax. $D(u)$ defines the Dawson function $D(u)=\exp \left(-u^{2}\right) \int_{0}^{u} \exp \left(t^{2}\right)$ $\mathrm{d} t$, which results from the averaging for an isotropic ensemble of platelets ${ }^{7}$ and exhibits the following asymptotic behavior: for $u \rightarrow \infty, 2 D(u) / u \rightarrow 1 / u^{2}$ and for $u \rightarrow 0, D(u) / u \rightarrow 1$. The scattering of the molecules in solution was added (eq 5 with an exponent $P=5 / 3$ ) giving an additional contribution to the intensity at high $Q$. The results are reported in Table 3 and shown in Figure 5 as solid lines describing the data measured at $0{ }^{\circ} \mathrm{C}$ for both contrast conditions. The disk radius is about $R$ $=3.2 \mu \mathrm{m}$ while the thickness and density of the polymer and
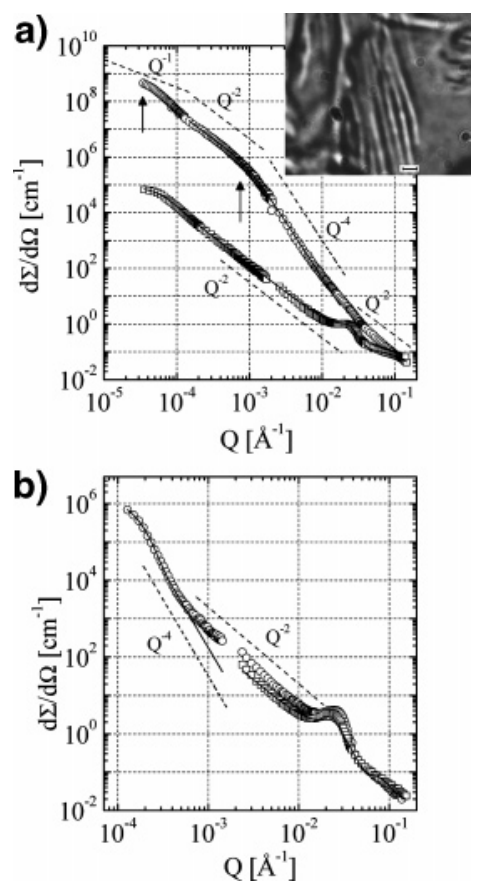

Figure 5. Scattering patterns under (a) wax (sample 3 ) and (b) polymer (sample 4) contrast from a mixed solution of $0.6 \%$ low $M_{\mathrm{w}}$ PEB-7.5 copolymer and $4 \% \mathrm{C}_{24}$ wax in decane at $0{ }^{\circ} \mathrm{C}$ (open squares) and -10 ${ }^{\circ} \mathrm{C}$ (open circles); dotted lines with the same meaning as in Figure 1; curves, fit of the data according to models (see text); arrows, the two Guinier regimes revealed by the wax scattering pattern at $-10^{\circ} \mathrm{C}$. The inset shows a micrograph of the aggregates formed in the same solution at $-10{ }^{\circ} \mathrm{C}$ (scale bar $\left.10 \mu \mathrm{m}\right)$

Table 3. Parameters of the Common Wax/Copolymer Aggregates Evaluated with Different Models As Described within the Text

\begin{tabular}{lcccccc}
\hline $\begin{array}{c}\text { sample/ } \\
\text { temp }\left[{ }^{\circ} \mathrm{C}\right]\end{array}$ & $\begin{array}{c}d_{\mathrm{p}} \\
{[\AA]}\end{array}$ & $\begin{array}{c}d_{\mathrm{w}} \\
{[\AA]}\end{array}$ & $\begin{array}{c}D^{* /} / \sigma_{\mathrm{D}} \\
{[\AA]}\end{array}$ & $\begin{array}{c}R_{\mathrm{disc}} \\
{[\mu \mathrm{m}]}\end{array}$ & $\begin{array}{c}\left(\Phi_{\mathrm{pol}}\right)^{\mathrm{agg}}(\%) \\
(\%)\end{array}$ & $\begin{array}{c}\left(\Phi_{\mathrm{wax}}\right)^{\mathrm{agg}} / \Phi_{\mathrm{wax}} \\
(\%)\end{array}$ \\
\hline $3 / 0$ & 110 & & $223 / 101$ & & 33 & \\
$4 / 0$ & & $32^{a}$ & $223 / 101$ & 3.2 & & 4 \\
$4 /-10$ & & 2300 & & $3.2^{b}$ & & 18 \\
$5 /-20$ & 81 & & $191 / 136$ & & 45 & \\
$6 /-20$ & & $32^{a}$ & $191 / 136$ & 3.1 & & 10 \\
$7 / 20$ & 155 & & $503 / 327$ & & 35 & \\
$8 / 20$ & & $45^{a}$ & $503 / 327$ & & & 20
\end{tabular}

${ }^{a}$ Fixed values for single stretched wax chains. ${ }^{b}$ Fixed values for same lateral extension of disks like that at $0{ }^{\circ} \mathrm{C}$.

wax layers agree with those found from the fit of the conventional SANS in terms of 2-dimensional aggregates, infinitely extended laterally. ${ }^{14}$ Because the correlation peak was found at the same $Q$ value as for the polymer self-assembling scattering patterns one can conclude that the primordial polymer needles, formed at higher temperatures than the wax $\mathrm{CP}$, influence significantly the later aggregation mechanism of the wax molecules and polymer chains in solution. At $-10{ }^{\circ} \mathrm{C}$ the scattering patterns of both components differ from one another. The polymer still retains the 2-dimensional correlated structures as demonstrated by the $Q^{-2}$ power law behavior at intermediate $Q$ and the correlation peak. The low $Q$ data measured by FSANS reveal the scattering from an additional structure, which is that of the large compact polymer crystals formed at much higher temperatures. The features of this structure were evaluated with the Beaucage model (eq 5 with a power law exponent $P=4$ ) and a comparison with the case of the polymer self-assembly shows that the addition of wax has only a minor effect on the morphology of these aggregates. The wax scattering pattern at $-10{ }^{\circ} \mathrm{C}$ reveals that the wax massively crystallizes and grows from thin platelets into large objects with sharp interfaces $\left(Q^{-4}\right.$ 
power law at higher $Q$ ). From an inspection of the low $Q$ data measured by FSANS $\left(10^{-4}\right.$ to $\left.2 \times 10^{-3} \AA^{-1}\right)$ and USANS (below $10^{-4} \AA^{-1}$ ) a coexistence of two structures showing different characteristic sizes can be identified (the vertical arrows indicate the observed Guinier regimes). The smaller shows a 2-dimensional morphology that is revealed by the $Q^{-2}$ power law regime identified within the $Q$ range covered by FSANS. The unified Beaucage equation ${ }^{24}$ for multiple structural levels and scattering data extending over many decades in $Q$

$$
\begin{aligned}
\frac{\mathrm{d} \Sigma}{\mathrm{d} \Omega}(Q)= & \sum_{i=1}^{n}\left(G_{i} \exp \left(-Q^{2} R_{\mathrm{g} i}{ }^{2} / 3\right)+\right. \\
& \left.B_{i} \exp \left(-Q^{2} R_{\mathrm{g}(i+1)}{ }^{2} / 3\right)\left\{\left[\operatorname{erf}\left(k_{i} Q R_{\mathrm{g} i} / \sqrt{6}\right)\right]^{3} / Q\right\}^{P}{ }_{i}\right)
\end{aligned}
$$

( $i=1$ represents the largest-size structural level) offers an approach of the lamellar or plate like aggregates in terms of two structural levels. The smallest pertains to the lamellar thickness and the Porod surface scattering regime. The largest pertains to the overall lamellar size and the 2-dimensional scaling regime between the lamellar thickness and overall size. A fit with eq 9 for the exponents $P_{1}=2$ and $P_{2}=4$ describes well the scattering pattern at intermediate $Q$ and allows an evaluation of the structural and density parameters of these aggregates (Table 3$)$. They are thick plates $(d=0.23 \mu \mathrm{m})$ which must result by the filling of the space between the thin correlated platelets identified at $0{ }^{\circ} \mathrm{C}$ with continuously growing wax crystallites. Thus, considering the new thicker structure as being made mostly by wax the "forward scattering" reveals a fraction of about $18 \%$ of all wax inside these aggregates. The additional scattering at low $Q$ indicates the formation of much larger aggregates whose characterization is difficult. This is done by analyzing scattering data where the Guinier regime is absent. A 1-dimensional morphology can be tentatively attributed to these aggregates via the assignment of $Q^{-1}$ for low $Q$. The neutron scattering findings for this later aggregation stage were complimented by the microscopy observations shown in Figure 2 and presented in the inset of Figure 5 where long needles with a thickness of several micrometers are observed.

As noted, it appears that the wax crystallites decorate the original polymer aggregates. The polymer needles form a primary morphology and show a longitudinal alternation of crystalline and amorphous sequences. The later wax crystallization is mediated apparently by the crystalline parts of these needles and the wax structures grow laterally also involving further polymer chains still in solution. In this way, large common wax and copolymer platelets develop around the needles as a secondary morphology. They contain most of the material while the primordial needles serve to template the lateral platelets in a correlated arrangement and contain only small amounts of polymers. This morphology is similar to the shish-kebab formed by crystallization induced flow orientation. The main requirement for the occurrence of this morphology is the generation of the fiber as a template for the later growing lateral platelets. In the case of polyethylene and cellulose, the fibers crystallize during flowing. ${ }^{25}$ In our case, the orientation mechanism is based upon the tendency of the polymer to aggregate into a graded one-dimensional structure prior to the inclusion of the paraffin. In the second step paraffin and polymer further cocrystallize in thin correlated platelets. A later aggregation step (at lower temperatures) leads to a thickening of the platelets by the crystallization of the wax surplus which grows from the 2-dimensional structures and fill the void volume. In this way, the correlation effect disappears from the scattering

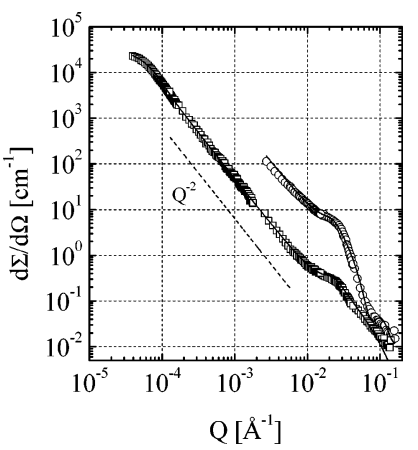

Figure 6. Scattering patterns under wax (open squares) and polymer (open circles ${ }^{14}$ ) contrast conditions from a mixed solution of $0.6 \%$ low $M_{\mathrm{w}}$ PEB-7.5 copolymer and $0.5 \% \mathrm{C}_{24}$ wax in decane (samples 5 and 6) at $-20{ }^{\circ} \mathrm{C}$; the solid curves represent model description of the experimental data (see text) while the dotted line indicate the power law regime identified at intermediate $Q$.

patterns collected under the wax contrast and thicker plates, as those revealed by the scattering at $-10{ }^{\circ} \mathrm{C}$, are formed. These become thicker and join leading to the appearance of the compact 1-dimensional tertiary structure. The modulation of the needle thickness that can be observed in the micrographs (the inset of Figure 5) is relatable with such a process yielding irregular needles. Their average thickness $(5-6 \mu \mathrm{m})$ agrees well with the lateral extension of the common wax-copolymer platelets revealed at $0{ }^{\circ} \mathrm{C}$. Thus, SANS and the microscopy observations are complementary and indicate that the aggregates observed evolve one from another with decreasing temperature and form a hierarchical morphology having multiple sized structures.

Figure 6 presents the scattering patterns from the mixed solution of low $M_{\mathrm{w}}$ PEB-7.5 copolymer and $0.5 \% \mathrm{C}_{24}$ wax in d-22 measured at $-20{ }^{\circ} \mathrm{C}$ under the polymer (sample 5) and wax contrast (sample 6). Large platelets formed and a correlated arrangement was observed (revealed by the wax scattering features which are very similar to those from sample 4 at 0 $\left.{ }^{\circ} \mathrm{C}\right)$. The geometrical and density parameters have been evaluated from the fit of the data for both contrast conditions in terms of an ensemble of finite extended disks forming a correlated arrangement (eqs 6-8). The results are reported in Table 3 and reveal almost the same lateral extension of the disks as in the case of sample 4 while the amounts of polymer and wax contained by the layers is significantly higher. The initial amount of wax in solution was very low and thus, the waxy crystals do not grow from the 2-dimensional structures into a bigger morphology. The large scale needles - the tertiary morphology mentioned in the case of $4 \%$ wax concentration-are mostly absent after the examination of the sample at $-20{ }^{\circ} \mathrm{C}$ via microscopy (Figure 7a) and the polymer effect in reducing the wax crystals to much smaller morphologies is obvious when the micrographs are compared with those from a pure wax solution (Figure 7b).

3.4. Crystallization of $C_{36}$ Wax from Solution in the Presence of High $M_{\mathrm{w}}$ PEB-7.5 Copolymer. The high $M_{\mathrm{w}}$ copolymer was synthesized only in the protonated state. Therefore, when is mixed with $\mathrm{C}_{36}$ wax in decane, the matching of the polymer scattering length density with that of the solvent for allowing the wax visible was possible only by using protonated decane (h-22). This combination allowed the wax to become visible but induced also a high incoherent background that dominated the scattering at high $Q$. Nevertheless, the wax formed big structure crystals in solution giving raise to a considerable increase of the scattering intensity at intermediate and low $Q$. This allowed a strong signal of the scattering features 

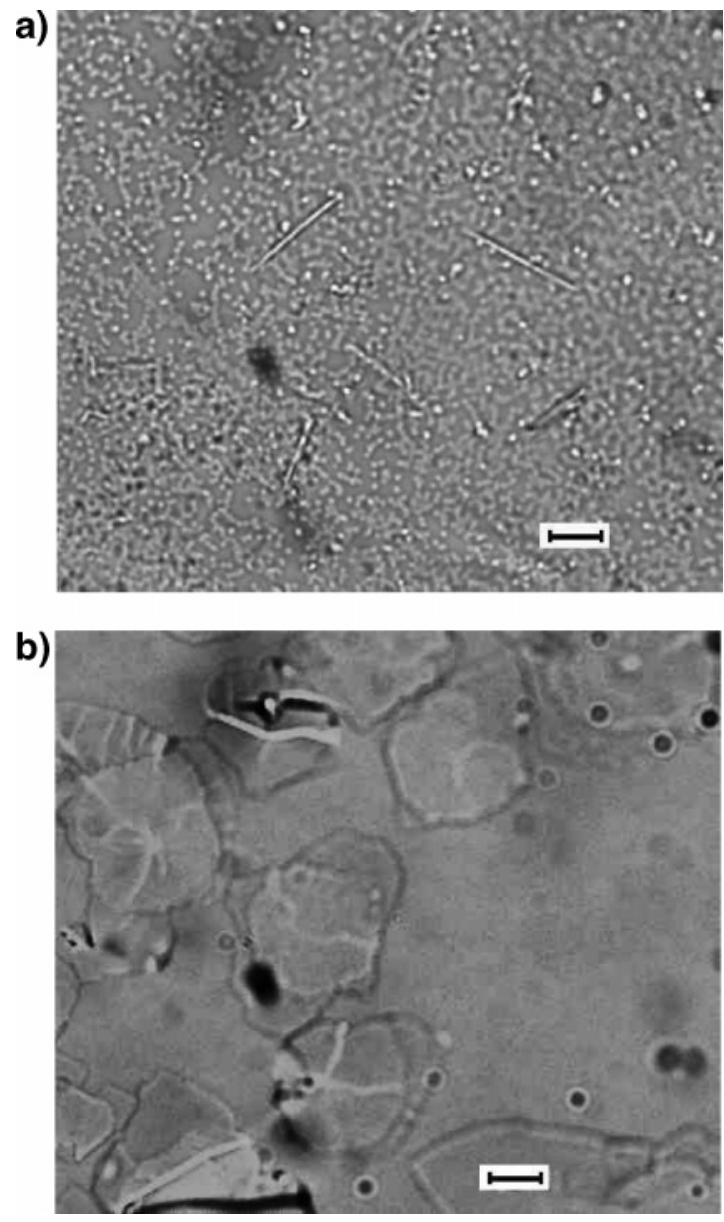

Figure 7. Optical micrographs at $-20{ }^{\circ} \mathrm{C}$ from decane solutions of mixture of $6 \mathrm{~K}$ PEB-7.5 and $0.5 \% \mathrm{C}_{24}$ wax (a) and of $0.5 \% \mathrm{C}_{24}$ wax (b). Scale bars: $30 \mu \mathrm{m}$.

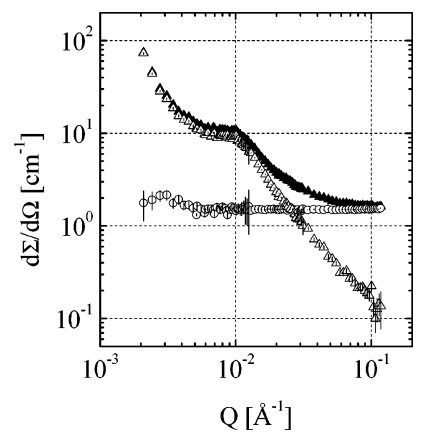

Figure 8. Scattering pattern under wax contrast from a mixed solution of $1 \%$ high $M_{\mathrm{w}}$ PEB-7.5 copolymer and $4 \% \mathrm{C}_{36}$ wax in decane (sample 7) at $40{ }^{\circ} \mathrm{C}$ in parallel with that of matched copolymer with protonated decane. The scattering from the wax aggregates (open triangles) is revealed after subtraction of the data for the matched solution (open circles) from those measured under the wax contrast (full triangles).

after subtraction of the matched solvent and polymer contribution. An example of the reliability of the data under the wax contrast at temperatures within the aggregation regime is given in Figure 8. It can be observed that the level of intensity under wax contrast is about 70-80 times higher than that of the matched polymer and solvent solution. Also, the scattering features are characteristic of wax aggregates while the scattering from the matched polymer and decane is completely flat. Figure 9a presents the polymer and wax scattering patterns measured by conventional SANS on a mixed solution of 30K PEB-7.5 copolymer and $\mathrm{C}_{36}$ wax in $\mathrm{d} 22$ (samples 7 and 8) at $40{ }^{\circ} \mathrm{C}$, within the common aggregation regime of this wax-copolymer
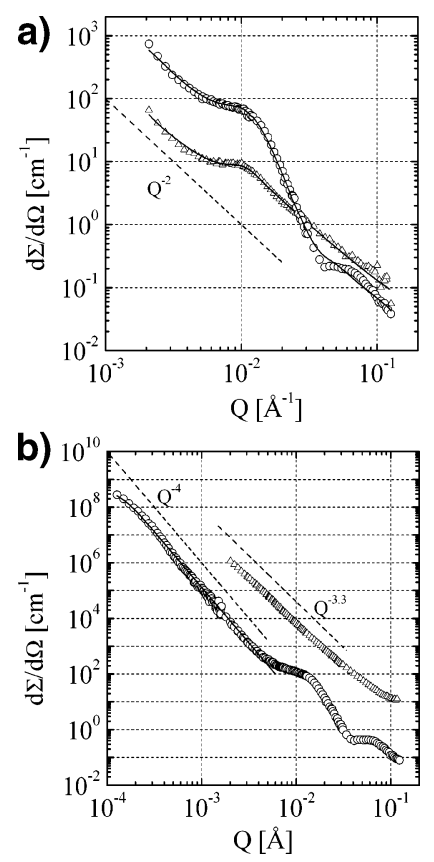

Figure 9. Scattering patterns under polymer (open circles, sample 6) and wax (open triangles, sample 7) contrast from a mixed solution of $1 \% 30 \mathrm{~K}$ PEB-7.5 copolymer and $4 \% \mathrm{C}_{36}$ wax in decane at two temperatures within the common aggregation regime: $40{ }^{\circ} \mathrm{C}$ (a) and $20{ }^{\circ} \mathrm{C}$ (b; wax data multiplied by a factor of 100 for reasons of clearness). The dotted lines have the same meaning as in Figure 1 while the solid curves represent the model description of the experimental data (see text).

combination. The same behavior as in the case of low $M_{\mathrm{w}}$ PEB7.5 mixed with $\mathrm{C}_{24}$ wax is observed. The $Q^{-2}$ power law and the peak presence at the same $Q$ as for the polymer selfassembling patterns ${ }^{14}$ prove a cocrystallization of the polymer and wax in thin correlated platelets which were mediated by the primordial 1-dimensional density modulated polymer structures. The fit of the data was performed again in terms of two embedded layers that form a correlated arrangement described by the paracrystalline structure factor (eqs 6-8). In this case infinite extended disks have been considered as long as their lateral size was not approached within the $Q$ range investigated. The evaluated structural and density parameters of the aggregates are reported in Table 3. Each disk consists of two embedded layers, a wax monolayer formed by single stretched wax chains $\left(d_{\mathrm{w}}=45 \AA\right)$ and a thicker polymer one. The disks are correlated over a distance $D^{*}$ showing a large smearing and contain about $35 \%$ of all the polymers and $20 \%$ of all the wax in solution. At lower temperatures the situation changes (Figure $9 b)$. As in the case of the low $M_{\mathrm{w}}$ PEB-7.5 mixed with $\mathrm{C}_{24}$ wax the polymer remains in the 2-dimensional arrangement shown by the presence of the correlation peak and the scattering features at high $Q$. Nevertheless, a $Q^{-4}$ power law behavior is shown at the low $Q$ of the SANS data and extends over a wide $Q$ range within the FSANS domain indicating the existence of additional large aggregates. These appear to differ from the typical large compact polymer objects observed in the polymer self-assembly situation (Figure 3). An interpretation of their features in terms of Beucage model (eq 5) for the exponent $P$ $=4$ provided a radius of gyration $R_{\mathrm{g}} \geq 2 \mu \mathrm{m}$ and a forward scattering $\mathrm{d} \Sigma(Q=0) / \mathrm{d} \Omega \cong 1.2 \times 10^{9} \mathrm{~cm}^{-1}$. As the morphology of these objects could not be established an interpretation of the forward scattering was not done. It seems that the changes at low $Q$ in the polymer scattering pattern are induced by the wax crystallization which, in this case, either influences the morphology of the large scale polymer objects or involves the 

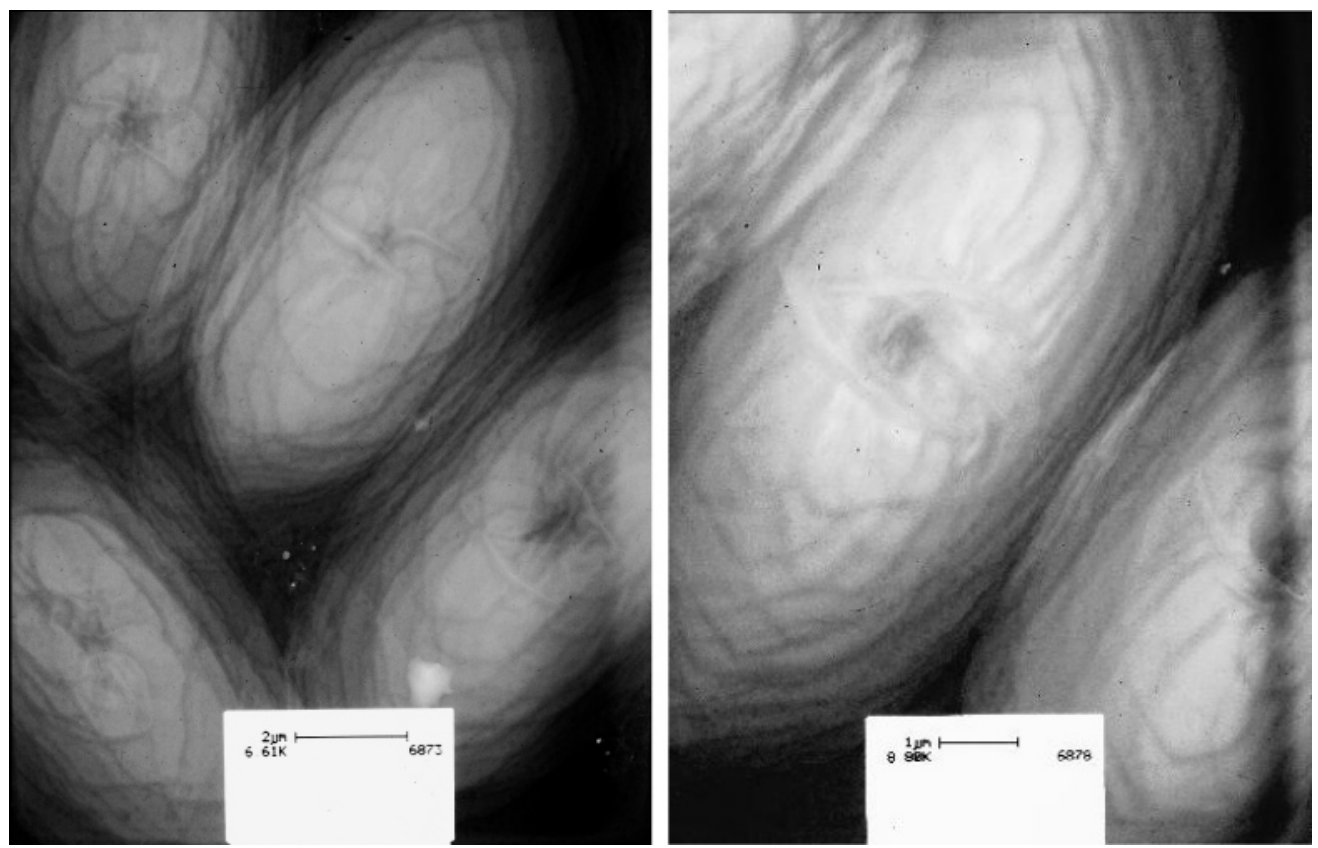

Figure 10. TEM images of the aggregates formed in a decane solution of $1 \% 30 \mathrm{~K}$ PEB-7.5 copolymer and $4 \% \mathrm{C}_{36}$ wax (scale bar: left, $2 \mu \mathrm{m}$; right, $1 \mu \mathrm{m})$.

polymer within some additional large scale structures. The wax scattering pattern at $20{ }^{\circ} \mathrm{C}$ shows that the wax crystallization buried the 2-dimensional wax structures since their scattering features - the correlation peak and the $Q^{-2}$ power law behaviordisappear. The scattering intensity behaves roughly like $Q^{-3.3}$ over almost the entire $Q$ range covered by the conventional SANS. FSANS measurements have not been performed as long as for the thinnest sample prepared $(0.2 \mathrm{~mm})$ the transmission was very low and thus, the multiple scattering were highly dominating. The SANS results prove that the wax crystals grow massively from the correlated platelets into large structures showing a surface fractal characteristic. Thus, the overall morphology of these large scale aggregates is difficult to resolve. The micrograph from Figure $4 \mathrm{~b}$ shows the presence of large needles similar to those found for low $M_{\mathrm{w}}$ copolymer and $\mathrm{C}_{24}$ wax. Additional large aggregates of an irregular shape are also visible in the upper part of the micrograph. Although the morphologies formed within this late aggregation stage cannot be fully characterized, both the scattering and the microscope observations prove that the same hierarchy of structures as in the case of the low $M_{\mathrm{w}}$ copolymer and $\mathrm{C}_{24}$ wax is principally formed. This is demonstrated by the TEM observations of the aggregates formed by the high $M_{\mathrm{w}} \mathrm{PEB}-7.5$ copolymer and $\mathrm{C}_{36}$ wax as isolated at room temperature from a decane solution. Results of these observations are shown in Figure 10: stacks of platelets arranged one in top of another and resembling a top view of a shish-kebab-like morphology are observed. The edges of the very large platelets and the central axis around which they have grown irregularly are well visible.

\section{Conclusions}

By a combination of microscopy observations in real space with SANS techniques, the morphologies formed in solutions of highly crystalline poly(ethylene-butene) random copolymers and in mixed solutions of these copolymers and paraffin waxes have been resolved in detail. Multilevel structures formed by graded aggregation processes have been characterized as regards the density and geometrical parameters. The polymer forms in solution two kinds of aggregates, large compact dumbbell-like objects and needlelike structures with modulated density distribution. When the polymers are mixed with wax molecules having a cloud point lower than the polymer self-assembling temperature, a hierarchical morphology showing multilevel structures is formed. First, the polymer 1-dimensional morphology showing density modulations occurs. Then, wax crystals are formed and nucleated by the crystalline nuclei of the polymer needles. The wax grows laterally in large platelets also involving further polymers still in solution. The primordial polymeric structure serves to template the lateral platelets in a correlated arrangement. Late aggregation leads to a massive crystallization of the wax surplus and formation of large compact 1-dimensional structure. Although both kinds of techniques revealed occurrence of large scale aggregates in the mixed polymerwax solutions an inspection of the largest structures sizes led to the conclusion that clogging of a $45 \mu \mathrm{m}$ filter - the standard size for defining the CFPP technical parameter-would not readily occur over a wide range of temperatures in comparison with the case of undoped wax solutions when much larger compact crystals are formed. This leads to the conclusion that the semicrystalline PEB-7.5 random copolymer could represent a highly efficient pour point depressant (and thus a low CFPP) particularly in the case of middle distillate fuels rich in short wax molecules. Recent work $^{26}$ has shown that crystallineamorphous copolymers can be made directly via the use of ethylene and $\alpha$-olefin monomers. A chain shuttling agent is used to transfer the growing chains between two catalysts having different monomer selectivities.

Acknowledgment. We acknowledge the assistance of Dr. M. Avramescu (Institute for Solid State Research-Soft Matter Group, Research Center Jülich) for obtaining the TEM images.

\section{References and Notes}

(1) Radlinski, A. P.; Barre, L.; Espinat, D. J. Mol. Cryst. 1996, 51, 383.

(2) Claudy, P.; Letoffe, J.-M.; Bonardi, B.; Vassiladis, D.; Damin, B. Fuel 1993, 72, 821

(3) Coutinho, J. A. P.; Dauphin, C.; Daridon, J. L. Fuel 2000, 79, 607.

(4) Abdallah, D. J.; Sirchio, S. A.; Weiss, R. G. Langmuir 2000, 16, 352.

(5) Kern, R.; Dassonville, R. J. Cryst. Growth 1992, 116, 191.

(6) The additive is a hydrogenated diblock copolymer where the initial segment is essentially 1,4 -butadiene $(\sim 2 \mathrm{~kg} / \mathrm{mol})$ followed by a $1,2-$ 
1,4-butadiene block. ( $\sim 5 \mathrm{~kg} / \mathrm{mol})$. Hydrogenation leads to the commercial additive. That product exhibits excellent nucleator activity in middle distillate fuels at concentrations as low as $50 \mathrm{ppm}$. Also see Goberdan, D.; Tack, R. D.; Lewtas, K.; McAleer, A. M.; Fetters, L. J.; Huang, J. S. US Patent 6,767,374, 27 July, 2004.

(7) Richter, D.; Schneiders, D.; Monkenbusch, M.; Willner, L.; Fetters, L. J.; Huang, J. S.; Lin, M.; Mortensen, K.; Farago, B. Macromolecules 1997, 30, 1053.

(8) Monkenbusch, M.; Schneiders, D.; Richter, D.; Willner, L.; Fetters, L. J.; Huang, J. S.; Lin, M. Physica B 2000, 276-278, 941.

(9) Leube, W.; Monkenbusch, M.; Schneiders, D.; Richter, D.; Adamson, D.; Fetters, L. J.; Dounis, P.; Lovegrove, R. Energy Fuels 2000, 14, 419.

(10) Schwahn, D.; Richter, D.; Wright, P. J.; Symon, C.; Fetters, L. J.; Lin, M. Macromolecules 2002, 35, 861.

(11) Schwahn, D.; Richter, D.; Lin, M.; Fetters, L. J. Macromolecules 2002 35,3762 .

(12) Ashbaugh, H. S.; Radulescu, A.; Prud'homme, R. K.; Schwahn, D.; Richter, D.; Fetters, L. J. Macromolecules 2002, 35, 7044

(13) Radulescu, A.; Schwahn, D.; Richter, D.; Fetters, L. J. J. Appl. Crystallogr. 2003, 36, 995.

(14) Radulescu, A.; Schwahn, D.; Monkenbusch, M.; Fetters, L. J.; Richter, D. J. Polym. Sci., B: Polym. Phys. 2004, 42, 3113.
(15) Guo, X.; Pethica, B. A.; Huang, J. S.; Prud'homme, R. K.; Adamson, D. A.; Fetters, L. J. Energy Fuels 2004, 18, 930.

(16) Morton, M.; Fetters, L. J. Rubber Chem. Technol. 1975, 48, 359.

(17) Asbaugh, H. S.; Fetters, L. J.; Adamson, D. H.; Prud'homme, R. K. J. Rheol. 2002, 46, 763

(18) Ashbaugh, H. S.; Guo, X.; Schwahn, D.; Prud'homme, R. K.; Richter, D.; Fetters, L. J. Energy Fuels 2005, 19, 138.

(19) www.neutronscattering.de/instruments.

(20) Kentzinger, E.; Dohmen, L.; Alefeld, B.; Ruecker, U.; Stellbrink, J.; Ioffe, A.; Richter, D.; Brueckel, T. Physica B 2004, 350, e779.

(21) Schwahn, D.; Yoo, M. H. Springer Proceedings in Physics; SpringerVerlag: Berlin, Heidelberg, Germany, New York, and Tokyo, 1986; Vol. 10, p 83

(22) Beaucage, G. J. Appl. Crystallogr. 1995, 28, 717.

(23) Schweins, R.; Huber, K. Macromol. Symp. 2004, 211, 25.

(24) Beaucage, G. J. Appl. Crystallogr. 1996, 29, 134.

(25) Ratta, V. Ph.D. Thesis, Virginia Polytechnic Institute and State University, Blacksburg, VA, 1999.

(26) Arriola, D. J.; Carnahan, E. M.; Hustad, P. D.; Kuhlman, R. L.; Wenzel, T. T.; Science 2006, 312, 714.

MA061104I 